Research Article

\title{
He-Plasma Jet Generation and Its Application for E. coli Sterilization
}

\author{
Tiejian Liu, ${ }^{1}$ Yuxuan Zeng $\mathbb{D}^{2},{ }^{2}$ Xin Xue, ${ }^{3}$ Yinyi Sui, ${ }^{3}$ Yingying Liang, ${ }^{3}$ Fushan Wang, \\ and Fada Feng $\mathbb{D}^{3,4}$ \\ ${ }^{1}$ Department of Neurosurgery, The Third Affiliated Hospital of Southern Medical University, Guangzhou 510630, China
${ }^{2}$ Shenzhen Engineering Center for the Fabrication of Two-Dimensional Atomic Crystals,
Shenzhen Institutes of Advanced Technology, Chinese Academy of Sciences, Shenzhen 518055, China
${ }^{3}$ School of Chemistry and Environment, Jiaying University, Meizhou 514015, China
${ }^{4}$ Guangdong Provincial Key Laboratory of Conservation and Precision Utilization of Characteristic Agricultural Resources in
Mountainous Areas, Jiaying University, Meizhou 514015, China
}

Correspondence should be addressed to Yuxuan Zeng; thomasez@126.com and Fada Feng; fadafeng@jyu.edu.cn

Received 11 October 2020; Revised 8 February 2021; Accepted 19 February 2021; Published 27 February 2021

Academic Editor: Jeongkwon Kim

Copyright (c) 2021 Tiejian Liu et al. This is an open access article distributed under the Creative Commons Attribution License, which permits unrestricted use, distribution, and reproduction in any medium, provided the original work is properly cited.

Atmospheric pressure plasma jet (APPJ) is a promising technique for the sterilization of pathogenic microorganisms in an ambient environment. In this work, a helium-APPJ was generated by double dielectric barrier discharge and applied to the sterilization of model microorganism in air and water. Discharge characteristics (including waveform and frequency of applied voltage), jet properties (such as feed gas flow rate, jet length, thermal effect, and optic emission spectra), and sterilization performance (in terms of clear/sterilized area, size of plaques, and sterilization efficiency) were investigated. Homogeneous helium plasma jet was generated in an energy-efficient way $(18 \mathrm{kHz}, 6 \mathrm{kV}, 0.08 \mathrm{~W})$ with a $19 \mathrm{~mm}$ jet and limited heating. The He-APPJ achieved good sterilization performances within very short treatment time (as short as $30 \mathrm{~s}$ ). For surface sterilization, the area of clear zone and size of the plaque were $1809 \mathrm{~mm}^{2}$ and $48 \mathrm{~mm}$, respectively, within 5 min treatment. For water sterilization, $99.8 \%$ sterilization efficiency was achieved within 5 min treatment. The optic emission spectra suggest that active species such as excited molecules, ions, and radicals were produced in the He-APPJ. The as-produced active species played important roles in the sterilization process.

\section{Introduction}

Microorganisms, including bacteria, fungi, archaea, and protozoa, were discovered by Leeuwenhoek in 1675. They exist in natural environment and are heavily involved in human activities. Many microorganisms are pathogenic and responsible for various diseases, such as skin diseases, tooth decay, respiratory infections, and gastrointestinal infections [1], endangering human health in global countries.

Traditional methods for the sterilization of pathogenic microorganisms include chemical, physical, and biological approaches [2-4]. Chemical methods use various chemical disinfectants that kill microorganisms efficiently. It is widely used in wound disinfection, water disinfection, and disinfection of epidemic areas. However, toxic disinfectant residues remain a problem for its further application [5]. Physical sterilization creates and utilizes extreme environment to destroy the physical structure of the microorganisms, by means of ultraviolet irradiation, heating, ionizing radiation, and microwave sterilization [6], and are often used in daily life or medical equipment disinfections. Biological sterilization is eco-friendly. However, the application is limited because of the strict requirements.

Nonthermal plasmas (NTPs) are characterized by the presence of electrons, ions, radicals, excited atoms, and molecules under ambient conditions [7]. In plasma, the energy of electrons can reach $1-20 \mathrm{eV}$ [8], being able to produce clusters of active species such as excited atoms, 
positive or negative ions, and radicals via the collision between gas molecules and energized electrons [9]. These active species, for instance, reactive nitrogen species (RNS) and reactive oxygen species (ROS), can destroy cell structures including cell membranes, proteins, and nucleic acids, leading to the inactivation or death of microorganisms $[10,11]$. In fact, RNS and ROS have been found to induce apoptosis in cancer cells [12]. Among different forms of NTP, the atmospheric pressure plasma jet (APPJ) is a promising technology for various applications, including modification of hydrophilic and chemically active materials [13], material surface etching [14], bacterial inactivation [15], skin disinfection [16], wound treatment [17], caries treatment [18], blood coagulation [19], and cancer cell apoptosis [20].

APPJ can be generated and powered by pulsed direct current (DC), alternating current (AC), radio frequency (RF), and microwave power sources [21, 22]. For generating AC-driven plasma jets, the dielectric barrier discharge (DBD) configuration is advantageous as it can work in a wide range of applied voltage under atmospheric pressure, while helium ( $\mathrm{He}$ ) and argon (Ar) are the mostly used as working gas [23]. In some cases, calculated amount of $\mathrm{O}_{2}$, $\mathrm{N}_{2}, \mathrm{H}_{2} \mathrm{O}$, and/or air can be mixed with $\mathrm{He}$ or Ar to generate peculiar active species in APPJ $[24,25]$. It is noted that APPJ can be generated more efficiently in $\mathrm{He}$, as the length of jet in $\mathrm{He}$ is usually longer than that in Ar under similar operational conditions. Besides, the He metastable has a higher energy and longer lifetime than the Ar counterparts [26]. Therefore, He-APPJ generated by DBD is very suitable for the production of active species and sterilization of microorganisms [27]. However, more works are still needed to optimize the configuration and operational conditions of He-DBD-APPJ, and more efforts are required to study the mechanism of sterilization process using APPJ, especially the plasma biological effects. Recently, a "plasma dose" defined by equivalent total oxidation potential (ETOP) is proposed to provide a new insight into the fundamentals of plasma medicine [28]. Based on the comprehensive definition of plasma dose, the biological effects of plasma under different conditions can be quantitatively compared and confirmed, which is very beneficial to the application of plasma in the medical field.

In the present work, a double dielectric barrier discharge (DDBD) reactor was used for the generation of He-plasma jet. The characteristics of He-plasma jet, such as voltage waveform, plasma power, length of plasma jet, temperature distribution in plasma, and optical emission spectra (OES), were investigated. The performance of surface and water sterilization was evaluated. Additionally, the mechanism of sterilization by He-plasma jet was discussed.

\section{Materials and Methods}

Figure 1 shows the experimental setup of this work. The He-plasma jet was generated by a DDBD reactor. The reactor consisted of two embed quartz tubes, with inner diameters of $3 \mathrm{~mm}$ and $1 \mathrm{~mm}$, respectively. He flows through the gap between two tubes. A copper foil was wrapped outside of the outer quartz tube, serving as the ground electrode. A copper rod with a diameter of $0.8 \mathrm{~mm}$ was installed in the axis of the inner tube, functioning as the high-voltage electrode. The distance between the inner electrode and the nozzle was $15 \mathrm{~mm}$. A high-frequency AC high-voltage power source was used to energize the reactor. The experiment was carried out at atmospheric pressure and room temperature. The flow rate of He was controlled by a mass-flow controller (MFC). The experimental system in this work is similar to our previous work that uses Ar plasma jet for sterilization [29].

The applied voltage was measured by a digital oscilloscope (DS1102E, Rigol, China) and a high-voltage probe (HVP) (P5104, Tektronix, USA). According to the needs of the experiment, the output voltage and frequency of the power supply are adjusted to about $2 \mathrm{kV}, 3 \mathrm{kV}, 4 \mathrm{kV}, 5 \mathrm{kV}$, and $6 \mathrm{kV}$ and $10 \mathrm{kHz}, 12 \mathrm{kHz}, 14 \mathrm{kHz}, 16 \mathrm{kHz}$, and $18 \mathrm{kHz}$, respectively. Photographs of the He-plasma jets were taken by a digital camera (EOS 7D, Canon, Japan). The temperature distribution of the He-plasma jet was measured by using an infrared camera (A35, FLIR, USA). The optic emission spectra of the ionized and excited species in the discharge zone were obtained using a multichannel spectrometer (Avantes, Netherlands).

The discharge power $P$ was online real-time measured by Q-U Lissajous method [30, 31]; the charge $Q$ flowing through the $\mathrm{DBD}$ reactor can be measured from the voltage drop $U_{c}$ across an external capacitance $C_{\text {ext }}(150 \mathrm{pF})$ according to the relation:

$$
Q=C_{\text {ext }} \times U_{c} .
$$

The energy per cycle $E$ and discharge power $P$ are calculated as follows:

$$
\begin{aligned}
& E=\oint U . i \mathrm{~d} t=\oint U \mathrm{~d} Q=\text { area of } Q-U \text { diagram, } \\
& P=f \times E,
\end{aligned}
$$

where $f$ is the applied frequency $(\mathrm{Hz})$.

E. coli (ATCC25922) was used as model microorganism to evaluate the sterilization efficiency. The number density was obtained by means of heterotrophic plate count method. Figure 2(a) shows a typical growth curve for E. coli. The bacterial culture had an OD600 of about $1.6 \mathrm{~A}$ over an $8 \mathrm{~h}$ incubation period. When the density of $E$. coli in the bacterial culture was $10^{7}-10^{9} \mathrm{cfu} / \mathrm{mL}$, it indicated that the growth of cell reached the log phase and the E. coli was most active. Figure 2(b) shows a typical image of E. coli in the LB medium. More details of the culturing and preparation of microorganism samples and of the sterilization experiments can be found in the supplementary material.

\section{Results and Discussion}

3.1. Plasma Jet Generation. Figure 3 shows the typical waveforms of applied voltage with a frequency of $10 \mathrm{kHz}, 14 \mathrm{kHz}$, and $18 \mathrm{kHz}$, respectively. The output of the high-frequency power source stably sustained the He-APPJ. Interestingly, when the frequency increased from $10 \mathrm{kHz}$ to 


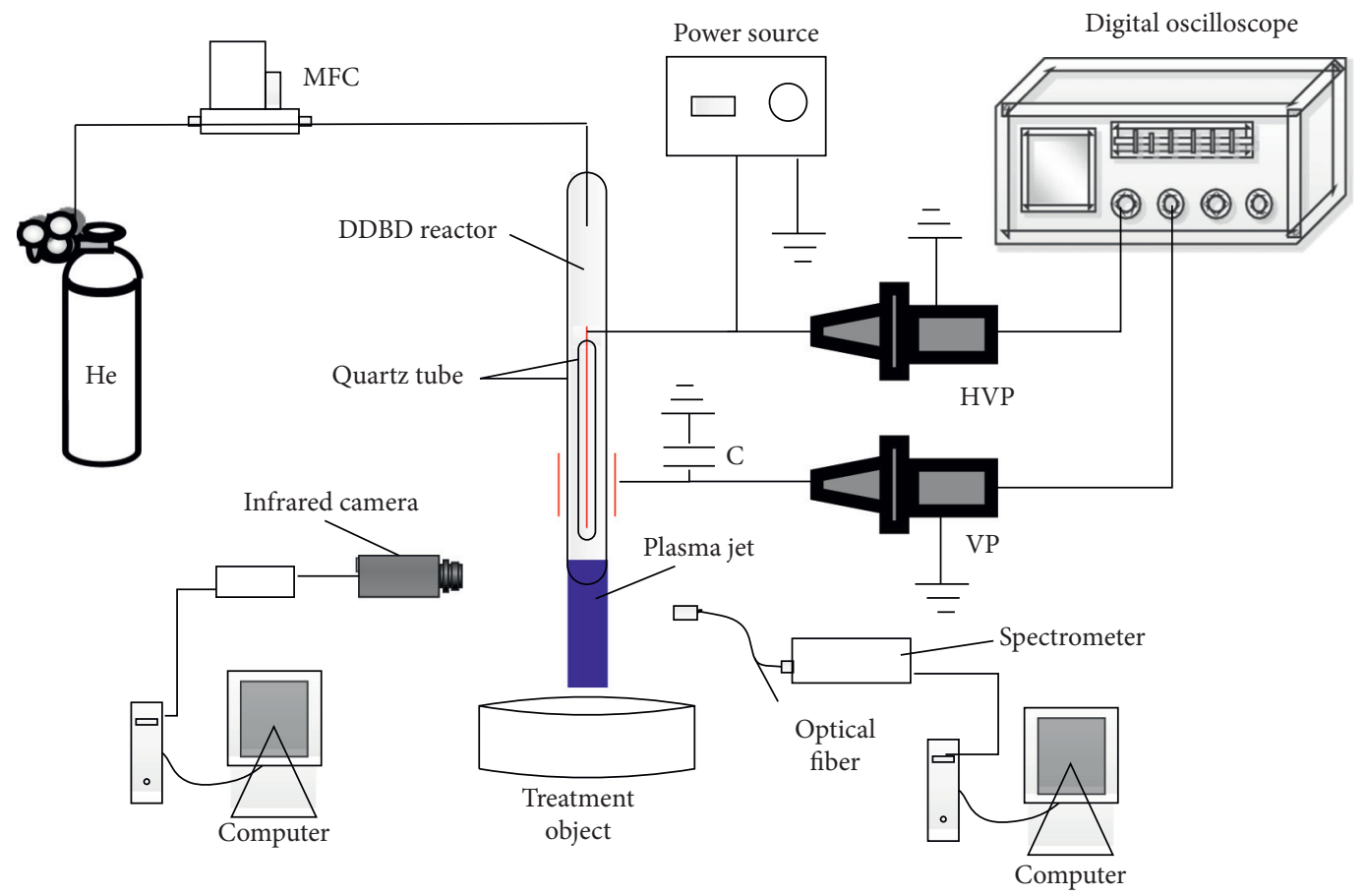

FIgURE 1: Schematic diagram of experimental setup.

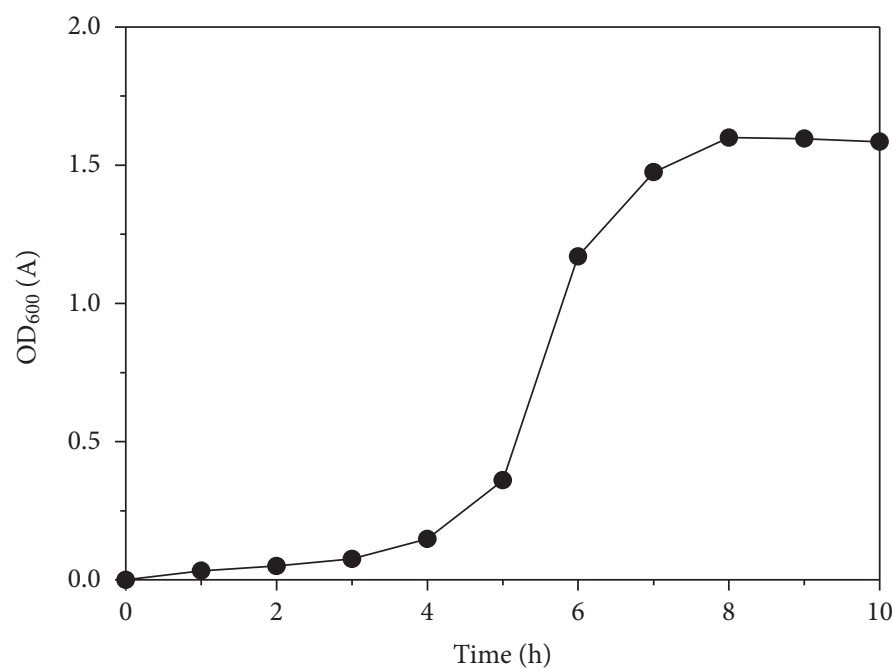

(a)

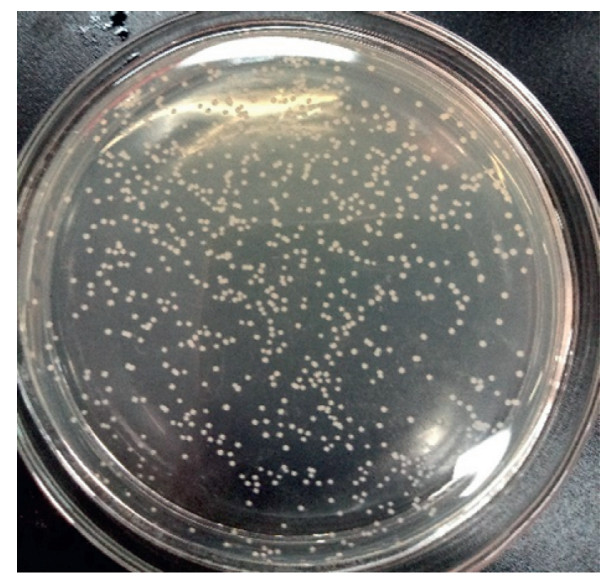

(b)

Figure 2: Typical (a) growth curve of E. coli and (b) image of E. coli in the LB medium.

$18 \mathrm{kHz}$, the amplitude of peak voltage decreased from $2 \mathrm{kV}$ to $1.6 \mathrm{kV}$. The frequency determined the number of discharges in unit time, while the peak voltage might determine power injection into plasma. Therefore, the efficiency was dependent on both factors [32]. Figure 4 shows a typical Lissajous Q-U figure of the DBD reactor. Based on the Lissajous figure, the discharge power can be calculated.

The power consumed by APPJ was very low in this work, implying that the APPJ was initiated and sustained in an energy-efficient way. Figure 5(a) shows the effect of applied voltage $(\mathrm{Vp}-\mathrm{p}, 18 \mathrm{kHz})$ on the discharge power of the $\mathrm{He}$ - plasma jet (power consumed by plasma jet). As the applied voltage increased, the discharge power of He-plasma jet gradually increased. For the applied voltages of $2 \mathrm{kV}, 5 \mathrm{kV}$, and $7 \mathrm{kV}$, the discharge power of plasma jet was $0.036 \mathrm{~W}$, $0.055 \mathrm{~W}$, and $0.092 \mathrm{~W}$, respectively. This result suggests that the average discharge current increased with the applied voltage. Figure 5(b) shows the dependence of plasma power on the feed flow rate of He. The frequency of applied voltage was $18 \mathrm{kHz}$. The discharge power of plasma jet increased from $0.032 \mathrm{~W}$ to $0.051 \mathrm{~W}$, as the gas flow rate increased from $0.5 \mathrm{~L} / \mathrm{min}$ to $3.5 \mathrm{~L} / \mathrm{min}$. However, further increase in flow 


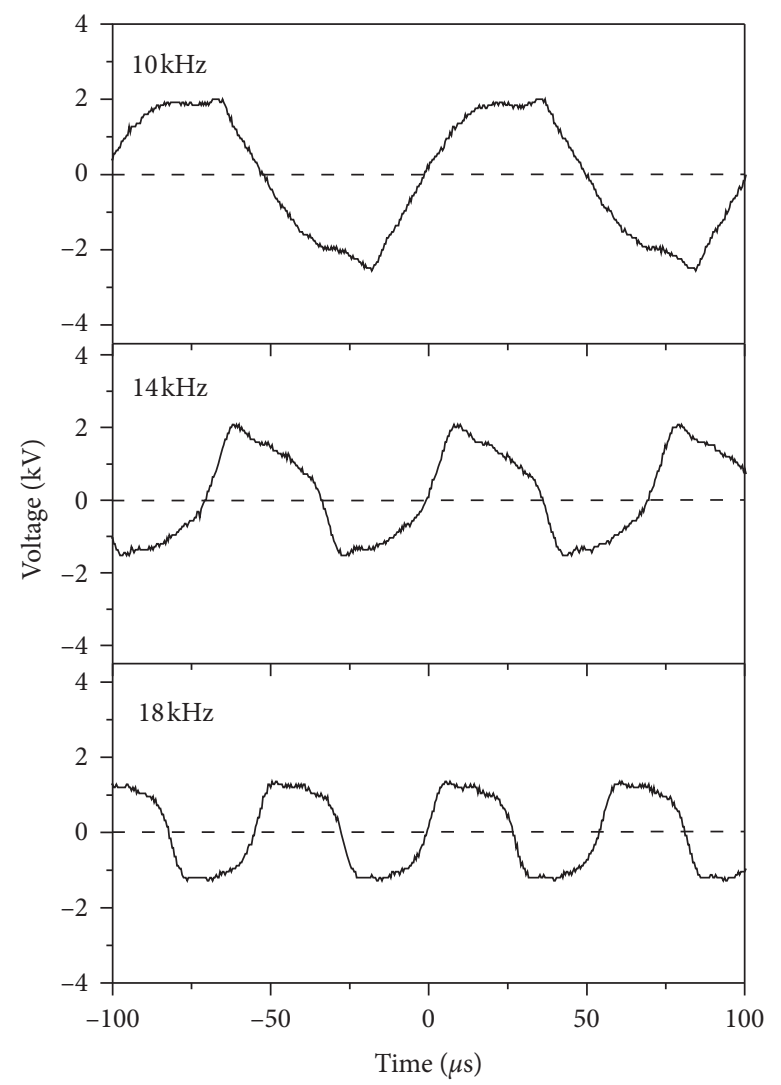

Figure 3: Typical voltage waveforms of the He-plasma jet with different frequencies.

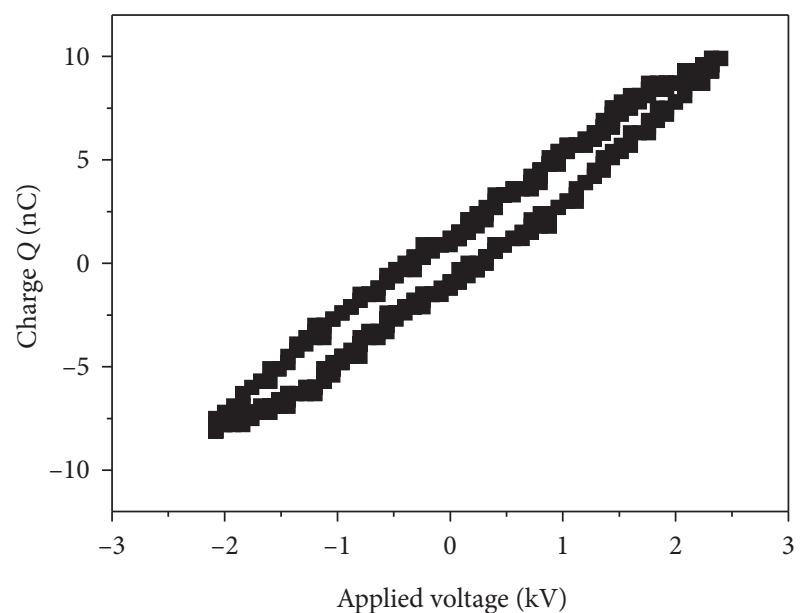

FIgURE 4: Typical Lissajous Q-U figures of the double dielectric barrier discharge.

rate resulted in the slight decrease in discharge power, suggesting that the discharge was disturbed when the flow rate was too high. Figure 5(c) shows the response of discharge power to the frequency of applied voltage. The flow rate of $\mathrm{He}$ was $1 \mathrm{~L} / \mathrm{min}$. As the frequency increased to $15 \mathrm{kHz}$, the discharge power of APPJ increased and reached a maximum of $0.095 \mathrm{~W}$. The discharge power quickly diminished when the frequency further increased. It is interesting that this phenomenon agrees with the results shown in Figure 3. Compared with the Ar plasma jet [29], the discharge power of the He-plasma jet is relatively small with similar conditions.

Figure 6 shows the photographs of He-APPJ generated under various conditions. Homogeneous plasma jet was generated by the DDBD configuration. The length of jet can be clearly observed. The length of He-plasma jet increased significantly along with the applied voltage (Figure 6(a)). For instance, when the applied voltage was $2 \mathrm{kV}$, the length of plasma jet was $3 \mathrm{~mm}$; on the contrary, when the applied voltage was $6 \mathrm{kV}$, the length of jet reached $19 \mathrm{~mm}$. This result agrees well with the evolution of jet power, as shown in Figure 5(a). The power of plasma jet increased at a higher voltage, suggesting that more energy was injected into the plasma; consequently, more He molecules were excited, and the plasma jet expanded. Figure 6(b) shows the photographs of He-plasma jet at different gas flow rates. The length of He-jet generally increased along with the gas flow rate and reached a maximum of $23 \mathrm{~mm}$ when the flow rate was $3.5 \mathrm{~L} / \mathrm{min}$. When the gas flow rate further increased to $4 \mathrm{~L} / \mathrm{min}$, the jet length slightly decreased. Notably, this result is consistent with the profile of plasma power, as shown in Figure 5(b). Figure 6(c) shows the change of He-plasma jet at different applied frequencies. An optimal frequency of applied voltage was observed for the longest He-APPJ. When the frequency was $14 \mathrm{kHz}$, the plasma jet stretched and reached its maximum of $16 \mathrm{~mm}$. 


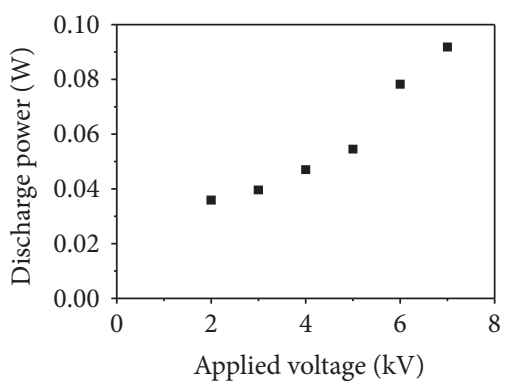

(a)

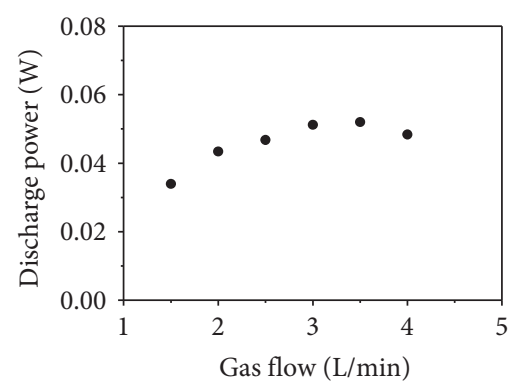

(b)

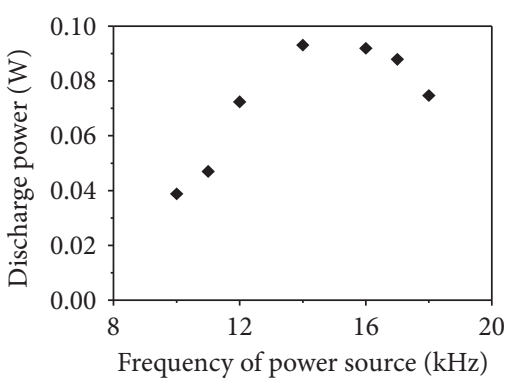

(c)

Figure 5: Effects of (a) applied voltage, (b) He gas flow rate, and (c) applied frequency on the discharge power.

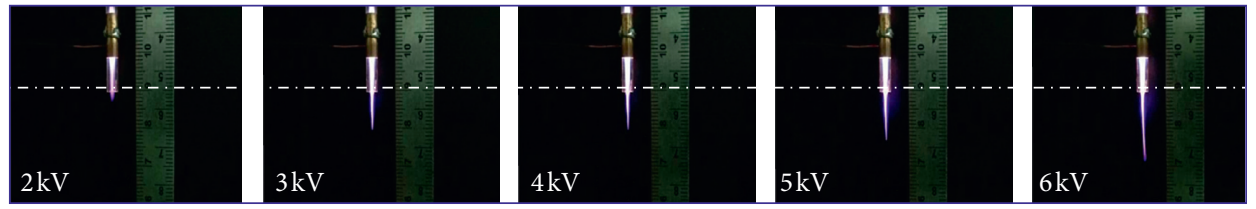

(a)
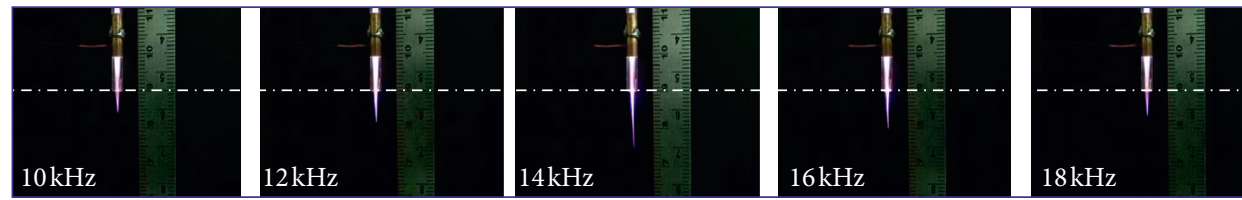

(b)
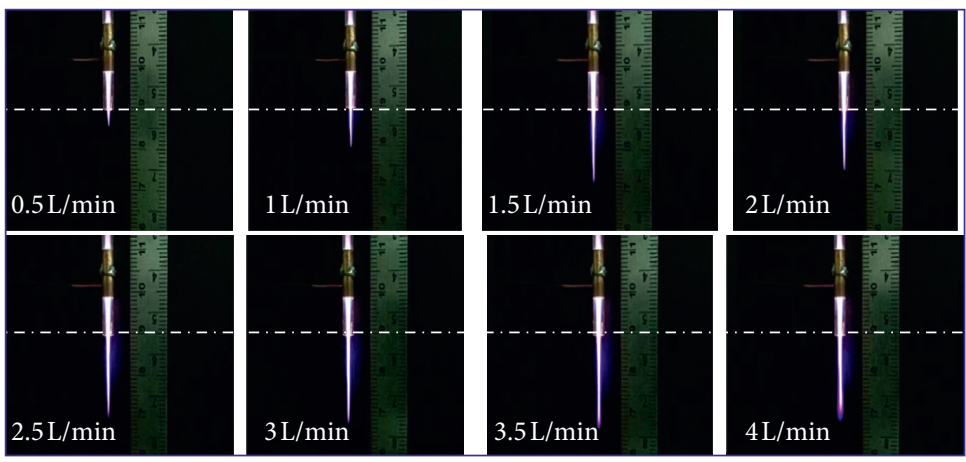

(c)

Figure 6: Photographs of He-plasma jets generated at different (a) applied voltages, (b) He flow rates, and (c) applied frequencies.

Interestingly, this result agrees well with the jet power data shown in Figure 5(c). All the results confirm that the discharge power determined the volume and length of APPJ, consequently determining the sterilization performance.

The temperature distribution in the plasma jet is very important for sterilizations. For instance, gas flow expands at high temperatures, changing the shape and length of plasma jet; discharge pattern changes at elevated temperatures; tissues and materials are damaged in contact with "hot" APPJ; and microorganisms are killed at sufficiently high temperatures. In this work, the temperature of APPJ was highly related to the amplitude of applied voltage. Figure 7 shows the temperature thermogram of the He-plasma jet at different applied voltages. Considering the plasma jet out of the DDBD outlet, the average and maximum temperatures were calculated. It is obvious that the average and maximum temperatures of the He-plasma jet were both close to ambient, which can be attributed to the low discharge power of the He-plasma jet. For example, at an applied voltage of $2 \mathrm{kV}, 4 \mathrm{kV}$, and $6 \mathrm{kV}$, the maximum temperature was $36.7^{\circ} \mathrm{C}$, $39^{\circ} \mathrm{C}$, and $42.1^{\circ} \mathrm{C}$, respectively. On the contrary, the applied voltage showed negligible effect on the average temperature of the He-APPJ. This result suggests that the plasma jet in this work can be used in the direct contact/treatment on tissues or other materials without the risk of thermal damage. This is especially valuable for tissue or skin sterilization, let alone APPJ is a chemical agent-free process. It 


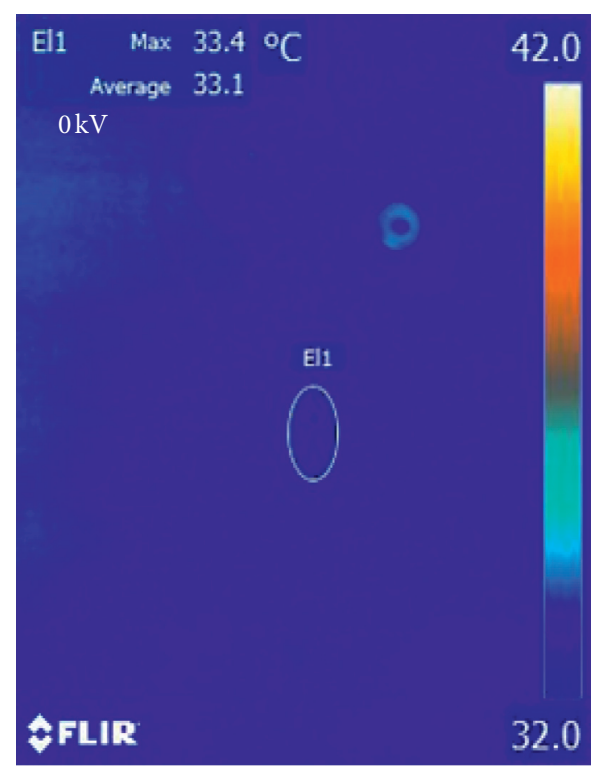

(a)

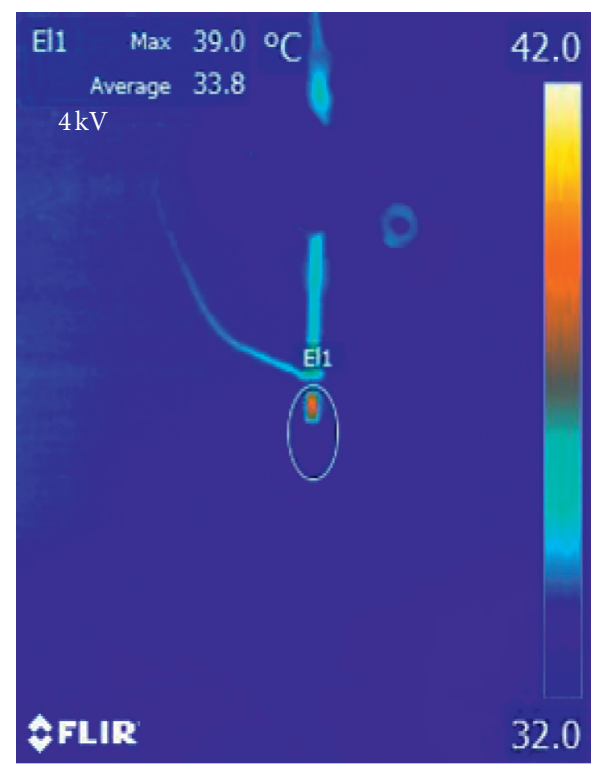

(c)

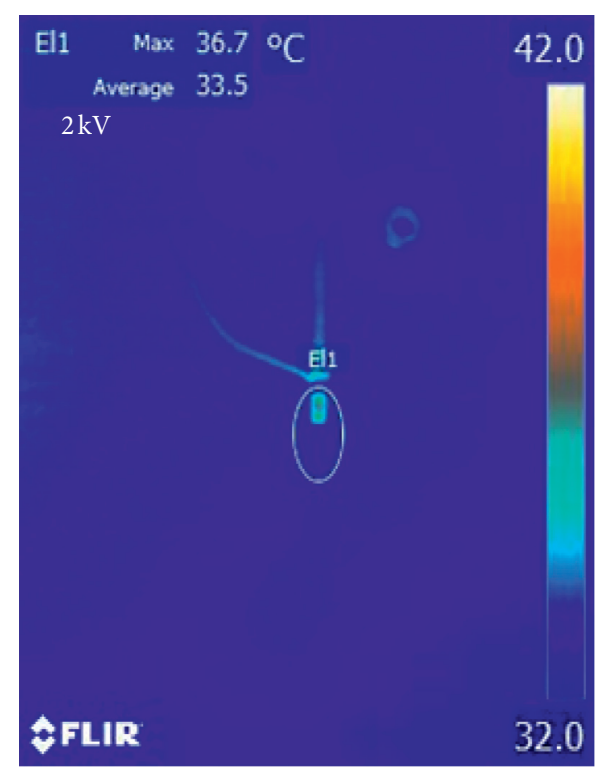

(b)

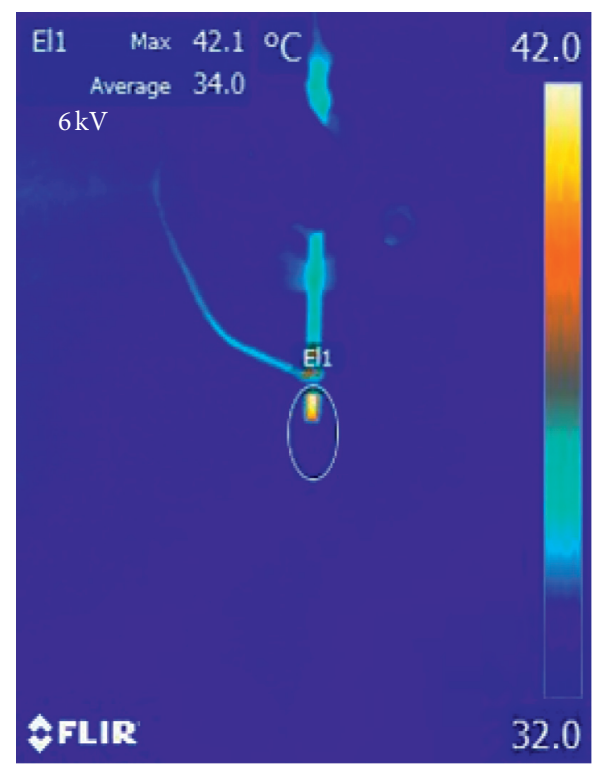

(d)

Figure 7: Temperature thermogram of the He-plasma jet at different applied voltages.

must be pointed out that the thermogram of APPJ indicates the negligible contribution of plasma heating during the sterilization process in this work.

3.2. Sterilization Using He-APPJ. Figure 8 shows the inhibited growth of model bacteria after He-plasma jet exposure $(18 \mathrm{kHz}, 6 \mathrm{kV}, 1.5 \mathrm{~L} / \mathrm{min})$, revealing the performance of surface sterilization using He-APPJ. Inhibition can be observed after a very short treatment period of $30 \mathrm{~s}$. The microorganisms in the inhibition zone were deactivated or killed by the He-APPJ. As the exposure time increased, the inhibition zones of bacteria (clear zone) expanded significantly (Figure $8(\mathrm{~b})$ ). For example, the area of clear zone increased from $28 \mathrm{~mm}^{2}$ to $1809 \mathrm{~mm}^{2}$ as the exposure time increased from $30 \mathrm{~s}$ to $5 \mathrm{~min}$ (Figure $8(\mathrm{a})$ ). The plaques are approximately $48 \mathrm{~mm}$ in diameter after $5 \mathrm{~min}$ of He-APPJ treatment.

Figure 9 shows the duration/period of He-APPJ treatment $(18 \mathrm{kHz}, 6 \mathrm{kV}, 1.5 \mathrm{~L} / \mathrm{min})$ and its effect on the efficiency of water sterilization. A high sterilization efficiency of $99.2 \%$ was achieved within a short treatment period of $1 \mathrm{~min}$. The efficiency further increased as the treatment duration increased. $99.8 \%$ of $E$. coli was sterilized after 5 min treatment. It can be explained that the active species produced by $\mathrm{He}$ APPJ entered the liquid phase and sterilized the bacteria, while for longer treatment durations, more active species were generated and transferred into water, enhancing the sterilization performance. 


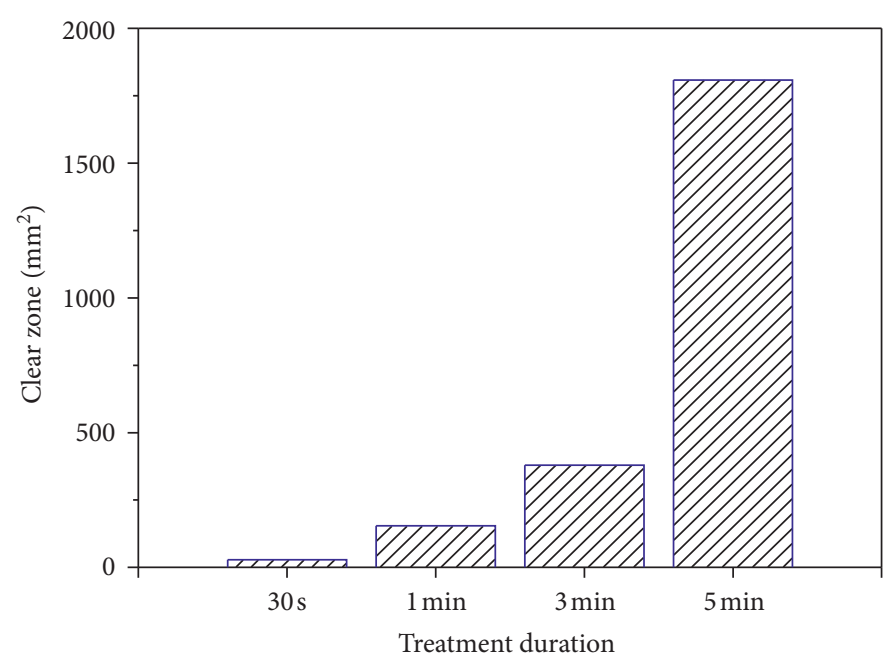

(a)

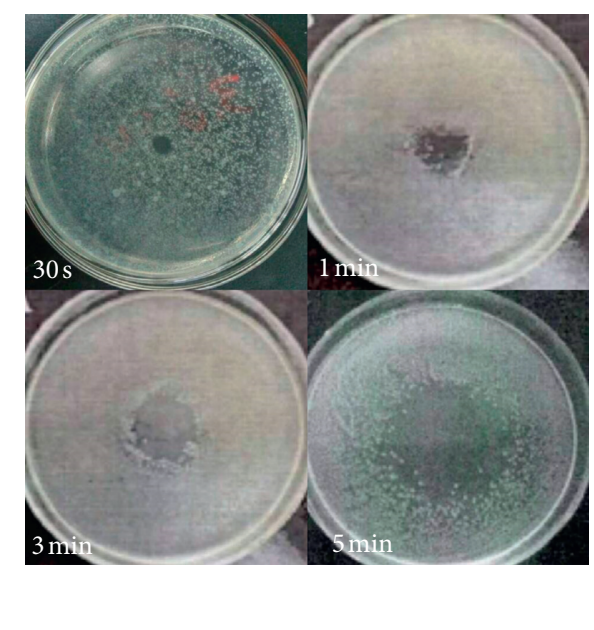

(b)

FIGURE 8: Growth inhibition of model bacteria after He-plasma jet exposure for different exposure times: (a) area of clear zone; (b) size of the plaque.

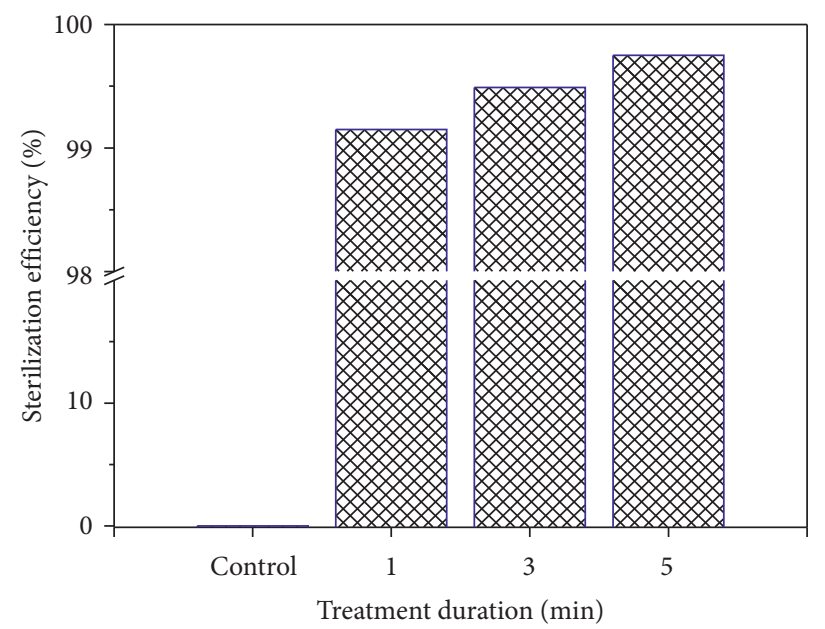

FIGURE 9: Effect of treatment duration on water sterilization.

3.3. Possible Mechanism of the Sterilization Process. When the He-plasma jet propagated in air, the energized electrons and excited $\mathrm{He}$ could collide with $\mathrm{N}_{2}, \mathrm{O}_{2}$, and $\mathrm{H}_{2} \mathrm{O}$ in air, subsequently generating a series of active species (e.g., $\mathrm{He}$ metastable, $\mathrm{N}_{2}$ metastable, $\mathrm{O}$ atoms, and $\mathrm{OH}$ radicals) $[33,34]$.

Figure 10 shows the optical emission spectra of the Heplasma jet. The excited molecules (such as He metastable and $\mathrm{N}_{2}$ metastable), ions $\left(\mathrm{N}_{2}{ }^{+}\right)$, and radicals ( $\mathrm{OH}$ and $\mathrm{O}$ radicals) can be identified from their vibrational emission bands [35]. The $\mathrm{N}_{2}{ }^{+}\left(\mathrm{B}^{2} \Sigma_{\mathrm{u}}\right)$ band is mainly related to the $\mathrm{N}_{2}{ }^{+}$ions produced by Penning ionization between $\mathrm{N}_{2}$ and $\mathrm{He}$ metastable [36]. Intensive emissions of $\mathrm{N}_{2}\left(\mathrm{C}_{3} \Pi_{\mathrm{u}}-\mathrm{B}^{3} \Pi_{\mathrm{g}}\right)$ can be clearly observed. The transition energy of corresponding species $(\nu=0-3)$ is $11.178,11.425,11.668$, and $11.908 \mathrm{eV}$, respectively [26]. The emission at $706.5 \mathrm{~nm}$ and $777.3 \mathrm{~nm}$ is related to the radiative transition of electrons from
$\mathrm{He}\left(3 \mathrm{~s}\left({ }^{3} \mathrm{~S}\right)\right) \quad(22.72 \mathrm{eV})$ and $\mathrm{O}\left(3 \mathrm{p}\left({ }^{5} \mathrm{P}\right)\right) \quad(10.74 \mathrm{eV})$ to $\mathrm{He}\left(2 \mathrm{p}\left({ }^{3} \mathrm{P}\right)\right)(20.96 \mathrm{eV})$ and $\mathrm{O}\left(3 \mathrm{~s}\left({ }^{5} \mathrm{~S}\right)\right)(9.14 \mathrm{eV})$, respectively $[37,38]$. OH $\left(\mathrm{A}^{2} \Sigma^{+}-\mathrm{X}^{2} \Pi\right)$ can be also identified from the spectra, and the corresponding transition energy is $4.2 \mathrm{eV}$ [26]. This spectra profile confirms the generation and participation of various active species in the sterilization process.

When the He-plasma jet was used to treat E. coli and other microorganisms in the agar media or water, excited metastable can collide with the cell and transfer energy to the groups and species on the cell membrane, resulting in deformation and malfunction of the membrane. In addition, the energized electrons and ions could collide with organic molecules on the cell and break the chemical bonds. Besides, the electrons and ions attached and accumulated on the membrane of cell, finally causing punctures. Moreover, the reactive oxidizing agents (e.g., ROS and RNS) produced in 


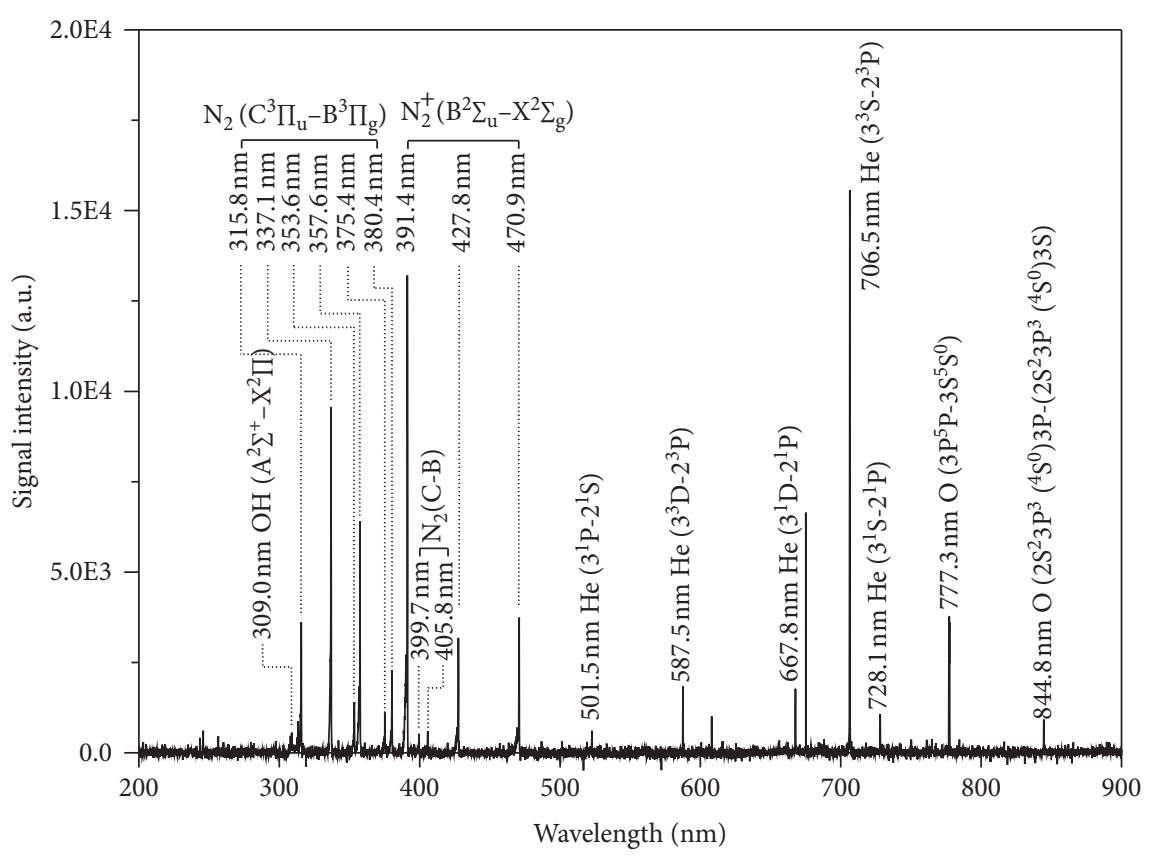

Figure 10: Optical emission spectra of the He-plasma jet.

APPJ [39] could react with and damage the cell or the contents. All these factors contributed to the sterilization. Especially, the ROS and RNS were proposed to play a crucial role in the inactivation of the bacteria.

It is very interesting that, based on the results shown in the emission spectra, the intensity of UV irradiation was very low in the He-plasma jet. This result agrees well with the literature from $\mathrm{Lu}$ et al. [40]. It is presented that the UV emission intensity can be as low as $0.05 \mathrm{~mW} / \mathrm{cm}^{2}$ [40]. Therefore, it can be concluded that the contribution of ultraviolet to sterilization was limited. Besides, as shown in Figure 7 , the contribution of heating/thermal effect to the sterilization was also very limited.

\section{Conclusions}

A homogeneous He-plasma jet was generated using a highvoltage AC power source and double dielectric barrier discharge at atmospheric pressure. Discharge characteristics (including waveform and frequency of applied voltage), jet properties (such as feed gas flow rate, jet length, thermal effect, and optic emission spectra), and sterilization performance (in terms of clear/sterilized area, size of plaques, and sterilization efficiency) were investigated. The length of He-plasma jet was highly related to the plasma power and increased along with the applied voltage. Optimal He flow rate and applied frequency were found for the generation of longest plasma jet. The heating of APPJ in this work was limited. For instance, the maximum temperature of the He-plasma jet only increased $5.4^{\circ} \mathrm{C}$ as the applied voltage increased from $2 \mathrm{kV}$ to $6 \mathrm{kV}$. Therefore, the He-APPJ has the potential for direct contact/ treatment of tissues or temperature-sensitive materials, without the risk of thermal damage. The He-APPJ in this work was also investigated for the sterilization of model microorganisms. Rapid sterilization and good performance were achieved. For surface sterilization, the area of inhibition zone and size of the plaque were $1809 \mathrm{~mm}^{2}$ and $48 \mathrm{~mm}$, respectively, within $5 \mathrm{~min}$ treatment. For water sterilization, high efficiency could be achieved within 1 min treatment and $99.8 \%$ sterilization efficiency was achieved within 5 min treatment. Finally, based on the optical emission spectra of the He-plasma jet, active species such as $\mathrm{He}$ metastable, $\mathrm{N}_{2}$ metastable, $\mathrm{N}_{2}{ }^{+}, \mathrm{OH}$, and $\mathrm{O}$ were identified. The possible contribution of the as-produced active species, plasma heating, and UV irradiation to the sterilization process was also discussed.

\section{Data Availability}

E. coli (ATCC25922) was firstly cultured in a prepared LuriaBertani (LB) medium at $310 \mathrm{~K}$ for $8-10$ hours. $10 \mathrm{~g} / \mathrm{L}$ of sodium chloride, $10 \mathrm{~g} / \mathrm{L}$ of peptone, and $5 \mathrm{~g} / \mathrm{L}$ of yeast extract were added into the medium. After culturing, the number density was $107-109 \mathrm{cfu} / \mathrm{mL}$. Then, the E. coli was harvested by centrifugation $(10000 \mathrm{rpm}, 2 \mathrm{~min})$ and suspended in deionized water. The surface sterilization efficiency of the He-plasma jet was determined based on the plaque size of E. coli on the solid agar plate. The agar plate had a diameter of $90 \mathrm{~mm}$. The distance between the outlet of DDBD reactor and the agar plate was about $15 \mathrm{~mm}$. The efficiency of water sterilization was quantified using an enzyme linked immunosorbent assay (ELISA) plate. The ELISA plate has 48 wells. Each well has a diameter of $6 \mathrm{~mm}$ and a depth of $11 \mathrm{~mm} .250 \mu \mathrm{L}$ of $E$. coli suspension was added into each well. The distance between the outlet of DDBD reactor and the ELISA plate was $8 \mathrm{~mm}$.

\section{Conflicts of Interest}

The authors declare that there are no conflicts of interest regarding the publication of this paper. 


\section{Acknowledgments}

This work was financially supported by the Natural Science Foundation of China (no. 21507043), Guangdong Natural Science Foundation (nos. 2018A0303130089 and 2016A030307009), Special Funds for the Cultivation of Guangdong College Students' Scientific and Technological Innovation ("Climbing Program" Special Funds) (no. pdjh2020b0555), and Undergraduate Innovation and Entrepreneurship Training Program (no. 202010582004X).

\section{References}

[1] E. Grawinski, "Human pathogenic microorganisms isolated from breeding fish and other water animals: a review," Medycyna Weterynaryjna, vol. 51, no. 7, pp. 383-386, 1995.

[2] X. Xue, R. Cheng, L. Shi, M. Kang, and Y. Zhu, "Removal of pathogenic microorganisms in water with nanomaterials: a review," Science \& Technology Review, vol. 34, pp. 1000-7857, 2016.

[3] L. H. Liegel, "Effects of sterilization procedures on the biological, chemical, and physical-properties of soils-a review," Turrialba, vol. 36, no. 1, pp. 11-19, 1986.

[4] M. A. de Moraes, R. F. Weska, and M. M. Beppu, "Effects of sterilization methods on the physical, chemical, and biological properties of silk fibroin membranes," Journal of Biomedical Materials Research Part B: Applied Biomaterials, vol. 102, no. 4, pp. 869-876, 2014.

[5] R. R. Ernst and J. E. Doyle, "Sterilization with gaseous ethylene oxide: a review of chemical and physical factors," Biotechnology and Bioengineering, vol. 10, no. 1, pp. 1-31, 1968.

[6] R. Lall, A. Sahu, A. Jaiswal, S. Kite, A. Sowmya, and M. Sainath, "Evaluation of various sterilization processes of orthodontic instruments using biological indicators and conventional swab test method: a comparative study," The Journal of Contemporary Dental Practice, vol. 19, no. 6, pp. 698-703, 2018.

[7] L. Tonks and I. Langmuir, "Oscillations in ionized gases," Physical Review, vol. 33, no. 2, pp. 195-210, 1929.

[8] J. Van Durme, J. Dewulf, C. Leys, and H. Van Langenhove, "Combining non-thermal plasma with heterogeneous catalysis in waste gas treatment: a review," Applied Catalysis B-Environmental, vol. 78, no. 3-4, pp. 324-333, 2008.

[9] N. Jiang, Y. Zhao, C. Qiu et al., "Enhanced catalytic performance of $\mathrm{CoOx}-\mathrm{CeO}_{2}$ for synergetic degradation of toluene in multistage sliding plasma system through response surface methodology (RSM)," Applied Catalysis B-Environmental, vol. 259, Article ID 118061, 2019.

[10] E. J. Szili, J. Oh, H. Fukuhara et al., "Modelling the helium plasma jet delivery of reactive species into a $3 \mathrm{D}$ cancer tumour," Plasma Sources Science \& Technology, vol. 27, no. 1, Article ID 014001, 2018.

[11] H. M. Joh, J. Y. Choi, S. J. Kim, T. H. Kang, and T. H. Chung, "Effects of the pulse width on the reactive species production and DNA damage in cancer cells exposed to atmospheric pressure microsecond-pulsed helium plasma jets," AIP Advances, vol. 7, Article ID 085106, 2017.

[12] S. J. Kim and T. H. Chung, "Plasma effects on the generation of reactive oxygen and nitrogen species in cancer cells in-vitro exposed by atmospheric pressure pulsed plasma jets," Applied Physics Letters, vol. 107, Article ID 063702, 2015.
[13] F. Duriyasart, M. Ohtani, J.-S. Oh, A. Hatta, and K. Kobiro, “A new approach to surface activation of porous nanomaterials using non-thermal helium atmospheric pressure plasma jet treatment," Chemical Communications, vol. 53, no. 50, pp. 6704-6707, 2017.

[14] T. Wang, B. Yang, X. Chen, X. Wang, C. Yang, and J. Liu, "Nonhomogeneous surface properties of parylene-C film etched by an atmospheric pressure $\mathrm{He} / \mathrm{O}_{2}$ micro-plasma jet in ambient air," Applied Surface Science, vol. 383, pp. 261-267, 2016.

[15] H. Xu, C. Chen, D. Liu et al., "The effect of gas additives on reactive species and bacterial inactivation by a helium plasma jet," Plasma Science and Technology, vol. 21, Article ID 115502, 2019.

[16] W. A. Rutala, M. F. Gergen, E. E. Sickbert-Bennett, and D. J. Weber, "Comparative evaluation of the microbicidal activity of low-temperature sterilization technologies to steam sterilization," Infection Control \& Hospital Epidemiology, vol. 41, no. 4, pp. 391-395, 2020.

[17] S. Pan, S. Zhang, and H. Chen, "Low temperature plasma promotes the healing of chronic wounds in diabetic mice," Journal of Physics D-Applied Physics, vol. 53, Article ID 185205, 2020.

[18] X. Lu, G. V. Naidis, M. Laroussi, S. Reuter, D. B. Graves, and K. Ostrikov, "Reactive species in non-equilibrium atmospheric-pressure plasmas: generation, transport, and biological effects," Physics Reports, vol. 630, pp. 1-84, 2016.

[19] K. Miyamoto, S. Ikehara, H. Takei et al., "Red blood cell coagulation induced by low-temperature plasma treatment," Archives of Biochemistry and Biophysics, vol. 605, pp. 95-101, 2016.

[20] Z. Chen, Q. Cui, C. Chen et al., "Inactivation of myeloma cancer cells by helium and argon plasma jets: the effect comparison and the key reactive species," Physics of Plasmas, vol. 25, Article ID 023508, 2018.

[21] R. Ono, "Optical diagnostics of reactive species in atmospheric-pressure nonthermal plasma," Journal of Physics D-Applied Physics, vol. 49, Article ID 083001, 2016.

[22] S. Wu, F. Wu, X. Liu, W. Mao, and C. Zhang, "A bipolar DC-driven touchable helium plasma jet operated in self-pulsed mode," IEEE Transactions On Plasma Science, vol. 46, no. 12, pp. 4091-4098, 2018.

[23] K. Ogawa, J. Oh, N. Gaur et al., "Modulating the concentrations of reactive oxygen and nitrogen species and oxygen in water with helium and argon gas and plasma jets," Japanese Journal of Applied Physics, vol. 58, Article ID SAAB01, 2019.

[24] H. M. Joh, E. J. Baek, S. J. Kim, and T. H. Chung, "Effects of the pulse width and oxygen admixture on the production of reactive species in gas- and liquid-phases exposed by bipolar microsecond-pulsed atmospheric pressure helium plasma jets," Physics of Plasmas, vol. 26, Article ID 053509, 2019.

[25] E. R. W. Van Doremaele, V. S. S. K. Kondeti, and P. J. Bruggeman, "Effect of plasma on gas flow and air concentration in the effluent of a pulsed cold atmospheric pressure helium plasma jet," Plasma Sources Science \& Technology, vol. 27, Article ID 095006, 2018.

[26] T. Shao, C. Zhang, R. Wang, Y. Zhou, Q. Xie, and Z. Fang, "Comparison of atmospheric-pressure He and Ar plasma jets driven by microsecond pulses," IEEE Transactions on Plasma Science, vol. 43, no. 3, pp. 726-732, 2015.

[27] G. Deng, Q. Jin, S. Yin et al., "Experimental study on bacteria disinfection using a pulsed cold plasma jet with helium/oxygen mixed gas," Plasma Science \& Technology, vol. 20, Article ID 115503, 2018. 
[28] H. Cheng, J. Xu, X. Li, D. Liu, and X. Lu, "On the dose of plasma medicine: equivalent total oxidation potential (ETOP)," Physics of Plasmas, vol. 27, Article ID 063514, 2020.

[29] F. Feng, X. He, Y. Liang et al., "Ar plasma jet generation and its application for water and surface sterilization," E3S Web of Conferences, vol. 78, p. 02020, 2019.

[30] X. Tu, H. J. Gallon, M. V. Twigg, P. A. Gorry, and J. C. Whitehead, "Dry reforming of methane over a Ni/ $\mathrm{Al}_{2} \mathrm{O}_{3}$ catalyst in a coaxial dielectric barrier discharge reactor," Journal of Physics D: Applied Physics, vol. 44, no. 27, Article ID 274007, 2011.

[31] F. Feng, Y. Zheng, X. Shen et al., "Characteristics of back corona discharge in a honeycomb catalyst and its application for treatment of volatile organic compounds," Environmental Science \& Technology, vol. 49, no. 11, pp. 6831-6837, 2015.

[32] N. Jiang, Y. Zhao, K. Shang et al., "Degradation of toluene by pulse-modulated multistage DBD plasma: key parameters optimization through response surface methodology (RSM) and degradation pathway analysis," Journal of Hazardous Materials, vol. 393, Article ID 122365, 2020.

[33] Y. Xia, N. Lu, J. Li, N. Jiang, K. Shang, and Y. Wu, "Combined steam and $\mathrm{CO}_{2}$ reforming of $\mathrm{CH}_{4}$ for syngas production in a gliding arc discharge plasma," Journal of $\mathrm{CO}_{2}$ Utilization, vol. 37, pp. 248-259, 2020.

[34] N. Jiang, L. Guo, C. Qiu et al., "Reactive species distribution characteristics and toluene destruction in the three-electrode DBD reactor energized by different pulsed modes," Chemical Engineering Journal, vol. 350, pp. 12-19, 2018.

[35] M. Ma, Y. Zhang, Y. Lv, and F. Sun, "The key reactive species in the bactericidal process of plasma activated water," Journal of Physics D: Applied Physics, vol. 53, no. 18, Article ID 185207, 2020.

[36] C. Li, J. H. Hsieh, and C. T. Yu, "Optical spectroscopy study for pulsed frequency powered atmospheric He plasma," Surface and Coatings Technology, vol. 353, pp. 316-323, 2018.

[37] G. Uchida, K. Takenaka, K. Kawabata, and Y. Setsuhara, "Influence of He gas flow rate on optical emission characteristics in atmospheric dielectric-barrier-discharge plasma jet," IEEE Transactions On Plasma Science, vol. 43, no. 3, pp. 737-744, 2015.

[38] R. Y. R. J. Kramida, A NIST Atomic Spectra Database (Ver. 5.6.1), National Institute of Standards and Technology, Gaithersburg, MD, USA, 2018.

[39] K. Takeda, H. Yamada, K. Ishikawa et al., "Systematic diagnostics of the electrical, optical, and physicochemical characteristics of low-temperature atmospheric-pressure helium plasma sources," Journal of Physics D: Applied Physics, vol. 52, no. 16, Article ID 165202, 2019.

[40] X. Lu, T. Ye, Y. Cao et al., "The roles of the various plasma agents in the inactivation of bacteria," Journal of Applied Physics, vol. 104, Article ID 053309, 2008. 